\section{Diagnosing idiopathic pulmonary fibrosis without a lung biopsy: honeycombing not required}

\author{
Robert Homer, ${ }^{1,2}$ David J Lederer ${ }^{3}$
}

Nearly 50 years ago, idiopathic interstitial pneumonias (IIPs) were defined by Liebow and Carrington based on the histological pattern present in the lung. ${ }^{12}$ Today, we consider histological patterns in the context of clinical and radiological information in order to diagnose a specific IIP, a paradigm shift initially introduced by a Consensus Statement published by the American Thoracic Society and European Respiratory Society in 2002 and developed further in 2011. ${ }^{3}$ With regard to idiopathic pulmonary fibrosis (IPF), this approach requires the presence of a usual interstitial pneumonia pattern (UIP), either on high resolution CT (HRCT) or histology or both, and the absence of any clinical history or morphological pattern that suggests another aetiology or another disease. The most recent iteration also introduced two new and related features. The first was a formal recognition that even in cases in which a biopsy was obtained, radiology had a role in modifying the interpretation of the pathological findings. In some cases, this meant that a definitive pathological diagnosis of IPF, once considered the gold standard, could be overridden by a negative radiological impression. In other cases, a diagnosis of IPF could be made even in the absence of definitive pathological evidence. The second major change was to introduce a multidisciplinary consensus conference as the mechanism for implementing the integration of pathology, radiology and clinical findings, such as in those cases just described in which there was substantial disagreement between pathology and radiology or those in which both evaluations were inconclusive. In many cases, however, pathology is still the definitive study especially in cases in which the biopsy reveals a diagnosis other than IPF.

\footnotetext{
${ }^{1}$ Department of Pathology, Yale University, New Haven, Connecticut, USA; ${ }^{2}$ Pathology and Laboratory Medicine Service, VA CT HeathCare System, West Haven, Connecticut, USA; ${ }^{3}$ Departments of Medicine and Epidemiology, Columbia University Medical Center, New York, New York, USA
}

Correspondence to Dr Robert Homer, Department of Pathology, Yale University, 310 Cedar St, New Haven, CT 06510, USA; robert.homer@yale.edu
In addition to providing some degree of standardisation, this approach has importantly provided a means to diagnose IPF without a surgical lung biopsy when the prebiopsy probability of IPF is sufficiently high (eg, an adult over the age of 50 years with a definite UIP pattern on HRCT and no alternative causes identified). ${ }^{4}$ Despite this success, however, a number of weaknesses in this overall approach have been identified, including only moderate reproducibility between centres, and the relatively large number of patients who do not meet the strict HRCT criteria (eg, lack of honeycombing leading to a 'possible UIP pattern' on HRCT) but who cannot be biopsied due to poor health or other reasons. $^{5-7}$

Several attempts to resolve this problem have explored relaxed radiological criteria. In patients with suspected IPF, a basilar predominant coarse reticular abnormality without honeycombing has been noted to be predictive of a pathological UIP pattern. ${ }^{8-10}$ The inclusion criteria for a recent IPF clinical trial permitted enrolment of patients with a possible UIP pattern on HRCT provided traction bronchiectasis was present on $\mathrm{CT}^{11}$ Moreover, a post hoc subgroup analysis of a large randomised clinical trial assessing the safety and efficacy of nintedanib showed that study participants with reticulation and traction bronchiectasis in the absence of honeycombing on HRCT had progression rates and responses to therapy that were similar to those with a UIP pattern. ${ }^{12}$ These last results are not completely surprising since the spectrum of anatomical abnormalities represented by traction bronchiectasis is quite common in UIP/IPF. ${ }^{13}$

Brownell and colleagues from University of California, San Francisco (UCSF) and Mayo Clinic extend these prior studies by developing and validating a clinical prediction rule to identify patients with a high likelihood of having a histological UIP pattern when a possible UIP pattern is present on HRCT in combination with additional clinical and radiological information. ${ }^{14}$ They used a discovery cohort from UCSF with a validation cohort from Mayo Clinic, Rochester. All HRCTs were re-evaluated by expert radiologists and reclassified as definite, possible or inconsistent with UIP. For scans read as a possible UIP pattern, the extent of traction bronchiectasis was scored. In the derivation cohort, a possible UIP pattern on HRCT had a $91 \%$ specificity for a histological UIP pattern (positive likelihood ratio 4.0). Using this cohort, the authors then developed a 'UIP score' ranging from 0 to 10 in which points are assigned to age, gender and extent of traction bronchiectasis in the setting of a possible UIP pattern on HRCT. A high UIP score, that is, in men age 60 years or greater and with extensive traction bronchiectasis but independent of the presence of honeycombing, increased the specificity of a possible UIP pattern on HRCT to $99.6 \%$ (positive likelihood ratio 45.7). Importantly, similar results were seen in the validation cohort, despite a very different overall prevalence of UIP/ IPF (29\% vs 67\%). This result means that these patients may avoid a biopsy to obtain a definitive diagnosis of UIP/IPF.

In interpreting the results of their study, the authors focus heavily on the impact of the prevalence of histological UIP patterns in the local population (or clinical practice setting). We propose expanding this approach further by considering individual patient pretest probability and not just population frequency. For example, a patient with a possible UIP pattern on HRCT along with inspiratory squeaks, water damage and mould in the home, and a few subtle areas of air trapping on expiratory imaging has a high likelihood of a diagnosis of chronic hypersensitivity pneumonitis and a very low probability of IPF. On the other hand, when alternative diagnoses are considered substantially less likely, the pretest probability for a histological UIP pattern may be quite high. Based on data from their derivation cohort, when the clinical likelihood of a histological UIP pattern is considered low (eg, $30 \%$ or less) a UIP score of 10 will yield a postevaluation probability of only $85 \%$, which may not be sufficient to avoid a biopsy, since a definite UIP pattern on HRCT has classically been associated with a $90 \%$ probability of a histological UIP pattern. ${ }^{15}$ For those with a 50\% pretest probability, a score of 10 will raise the post-test probability to $90 \%$, perhaps avoiding a biopsy. For those considered more likely to have a histological UIP pattern (eg, 70\% pretest probability), a score of only 6 is required to raise the post-test probability to $90 \%$ or more.

Using the clinical prediction rule, therefore, may yield post-test probability exceeding $90 \%$ in some clinical settings, 
perhaps avoiding a surgical lung biopsy, which can carry a risk of mortality of roughly $2 \%$ with additional risk depending on age, comorbidities, type of surgery and underlying diagnosis. ${ }^{16}$ Risk is particularly elevated when biopsy is done under non-elective conditions, emphasising that if biopsy is to be done, it should be done before the patient acutely deteriorates of possible. Therefore, the authors' prediction rule may have a substantial impact on reducing biopsy-related morbidity and mortality and perhaps on healthcare costs during the diagnostic evaluation of adults with interstitial lung disease. In those too ill to undergo biopsy, this clinical prediction rule should also grant access to FDA-approved therapies for IPF, and perhaps to enrolment in clinical trials for IPF.

While clinical and radiological diagnostic methods are of great use, other noninvasive approaches are also on the horizon. ${ }^{6}$ Interobserver agreement for a UIP pattern on HRCT is, at best, moderate. $^{7}$ It seems likely that automated texture analysis may improve on this result, and that machine-based recognition may one day help reduce this source of diagnostic variability and consequent diagnostic inaccuracy. ${ }^{17}$ We eagerly await clinical prediction models that incorporate genetic polymorphisms, serum protein and miRNA biomarkers, and tissue-based genomic signatures based on bronchoscopy rather than surgery. ${ }^{6}{ }^{18-20}$ Until then, we believe there is now nearly sufficient evidence to implement the authors' UIP Score model. While some might wish for a second independent study, if such a study is not forthcoming, upcoming modifications to the American Thoracic Society/European Respiratory Society/ Japanese Respiratory Society/Asociacion Latinamericana de Torax (Latin American Thoracic Association) (ATS/ERS/JRS/ ALAT) Clinical Practice Guidelines for the
Diagnosis of IPF should strongly consider incorporating this risk score into new recommendations for clinical practice.

Twitter Follow David Lederer @davidlederer

Competing interests None declared.

Provenance and peer review Commissioned; externally peer reviewed.

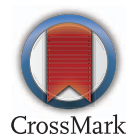

To cite Homer R, Lederer DJ. Thorax 2017;72:391-392.

Published Online First 9 March 2017

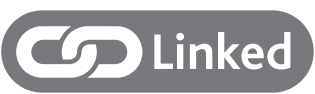

- http://dx.doi.org/10.1136/thoraxjnl-2016-209671

Thorax 2017:72:391-392.

doi:10.1136/thoraxjnl-2016-209831

\section{REFERENCES}

1 Liebow AA. The definition and classification of interstitial pneumonias in human pathology. Prog Resp Res 1974;8:1-33.

2 Liebow AA, Carrington CB. The interstitial pneumonias. In: Simon M, Potchen EJ, LeMay M, eds. Frontiers of pulmonary radiology. New York: Grune and Stratton, 1969:102-41.

3 Raghu G, Collard HR, Egan JJ, et al. An official ATS/ ERS/JRS/ALAT statement: idiopathic pulmonary fibrosis: evidence-based guidelines for diagnosis and management. Am J Respir Crit Care Med 2011;183:788-824.

4 Wells AU. The revised ATS/ERS/JRS/ALAT diagnostic criteria for idiopathic pulmonary fibrosis (IPF)practical implications. Respir Res 2013;14(Suppl 1):S2.

5 Wells AU. Any fool can make a rule and any fool will mind it. BMC Med 2016;14:23.

6 Martinez FJ, Chisholm A, Collard HR, et al. The diagnosis of idiopathic pulmonary fibrosis: current and future approaches. Lancet Respir Med 2017;5:61-71.

7 Walsh SL, Calandriello L, Sverzellati N, et al. Interobserver agreement for the ATS/ERS/JRS/ALAT criteria for a UIP pattern on CT. Thorax 2016;71:45-51.

8 Chung JH, Chawla A, Pelijto AL, et al. CT scan findings of probable usual interstitial pneumonitis have a high predictive value for histologic usual interstitial pneumonitis. Chest 2015;147:450-9.
9 Gruden JF, Panse PM, Leslie KO, et al. UIP diagnosed at surgical lung biopsy, 2000-2009: HRCT patterns and proposed classification system. AJR Am J Roentgenol 2013;200:W458-67.

10 Raghu G, Lynch D, Godwin JD, et al. Diagnosis of idiopathic pulmonary fibrosis with high-resolution CT in patients with little or no radiological evidence of honeycombing: secondary analysis of a randomised, controlled trial. Lancet Respir Med 2014:2:277-84.

11 Richeldi L, du Bois RM, Raghu G, et al. Efficacy and safety of nintedanib in idiopathic pulmonary fibrosis. N Engl J Med 2014;370:2071-82.

12 Raghu G, Wells AU, Nicholson AG, et al. Effect of nintedanib in subgroups of idiopathic pulmonary fibrosis by diagnostic criteria. Am J Respir Crit Care Med 2017;195:78-85.

13 Piciucchi S, Tomassetti S, Ravaglia C, et al. From "traction bronchiectasis" to honeycombing in idiopathic pulmonary fibrosis: a spectrum of bronchiolar remodeling also in radiology? BMC Pulm Med 2016;16:87.

14 Brownell R, Moua T, Henry TS, et al. The use of pretest probability increases the value of highresolution $\mathrm{CT}$ in diagnosing usual interstitial pneumonia. Thorax 2017;72:424-9.

15 Hunninghake GW, Zimmerman MB, Schwartz DA, et al. Utility of a lung biopsy for the diagnosis of idiopathic pulmonary fibrosis. Am J Respir Crit Care Med 2001;164:193-6.

16 Hutchinson JP, Fogarty AW, McKeever TM, et al. In-Hospital mortality after surgical lung biopsy for interstitial lung disease in the United States. 2000 to 2011. Am J Respir Crit Care Med 2016;193:1161-7.

17 Boehm HF, Fink C, Attenberger U, et al. Automated classification of normal and pathologic pulmonary tissue by topological texture features extracted from multi-detector CT in 3D. Eur Radiol 2008;18:2745-55.

18 White ES, Xia M, Murray $S$, et al. Plasma surfactant protein-D, matrix metalloproteinase-7, and osteopontin index distinguishes idiopathic pulmonary fibrosis from other idiopathic interstitial pneumonias. Am J Respir Crit Care Med 2016;194:1242-51.

19 Jenkins RG, Simpson JK, Saini G, et al. Longitudinal change in collagen degradation biomarkers in idiopathic pulmonary fibrosis: an analysis from the prospective, multicentre PROFILE study. Lancet Respir Med 2015;3:462-72.

20 Kim SY, Diggans J, Pankratz D, et al. Classification of usual interstitial pneumonia in patients with interstitial lung disease: assessment of a machine learning approach using high-dimensional transcriptional data. Lancet Respir Med 2015;3:473-82. 